\title{
RELACIONES OCTATÓNICAS EN EL PRIMER MOVIMIENTO DE LA SONATA PARA CLARINETE Y PIANO DE BENJAMÍN GUTIÉRREZ
}

\author{
Fernando Zúñiga Chanto*
}

\begin{abstract}
RESUMEN
El lenguaje utilizado por Benjamín Gutiérrez en su Sonata para Clarinete y Piano responde a la época en la cual el compositor escribió la obra. La evasión de la tonalidad se lleva a cabo por medio de la utilización de materiales de carácter simétrico, y esto se va a explicar por medio del análisis de la exposición del primer movimiento de la obra, utilizando la teoría de conjuntos para determinar los materiales sonoros. La estructura formal se ve afectada por el lenguaje utilizado por el compositor, y una reinterpretación de las estructuras formales debe tomarse en cuenta para poder analizar esta obra.

Palabras clave: Análisis, Sonata, Clarinete, Gutiérrez Benjamín.
\end{abstract}

\begin{abstract}
The musical language employed by Benjamin Gutierrez in his Sonata for Clarinet and Piano responds the the time in which the composer wrote the piece. The evasion of tonality is done by means of the use of symmetric materials, and this is going to be explained with the analysis of the exposition of the first movement of the piece, using set theory to determine the main sonic materials. The formal structure is affected by the language used by the composer, and a reinterpretation of the formal structures has to be taking into account to be able to analyze this piece.
\end{abstract}

Key Words: Analysis, Sonata, Clarinet, Gutiérrez Benjamín.

\section{Relaciones octatónicas en el primer movimiento de la Sonata para Clarinete y Piano de Benjamín Gutiérrez}

\section{Sonata para Clarinete and Piano}

Para entender la importancia de esta obra dentro del contexto de la obra de Benjamín Gutiérrez y del contexto de la música costarricense, se deben tomar varios aspectos en cuenta. Gutiérrez es parte de una generación de compositores que, tras establecer su nombre y estilo en Costa Rica, parten a realizar estudios en el exterior, tal como Julio Mata, Alejandro Monestel, Julio Fonseca, Bernal Flores, Carlos Enrique Vargas, los cuales asimilan las corrientes composicionales y estilísticas de la época, para evolucionar en un nuevo estilo, y son factores determinantes en la evolución de la composición en Costa Rica en la segunda mitad del siglo XX.

La Sonata para Clarinete y Piano fue terminada en el año 1959, y está dedicada

* $\quad$ Profesor de la Escuela de Música, Universidad de Costa Rica.

Recepción: 14/02/12. Aceptación:20/02/13. 
al clarinetista Sherman Friedland. Esta pieza fue escrita durante el período de estudios del compositor en New England Conservatory. Durante este período en la historia de la música, era algo común para los estudiantes de composición adoptar sistemas de composición tales como el dodecafonismo o el serialismo, tal y como lo mencionan los siguientes autores:

\begin{abstract}
Durante los años 1950, el serialismo estaba subiendo hacia un domino académico, y Coplanesque Americana estaba cayendo en desgracia ${ }^{1}$. No ser un serialista en la costa este de los Estados Unidos en los años 60 s era como no ser un católico en Roma en el siglo trece. Era la cosa respetable de hacer, aunque fuera sólamente una vez ${ }^{2}$. Tratar de escribir música tonal en un lugar como Columbia University en los 60s y 70s era como ser un disidente en Praga durante el mismo período, con consecuencias profesionales similares ${ }^{3}$.
\end{abstract}

Como las citas anteriores lo demuestran, esa época en Estados Unidos fue de un gran apogeo para la música atonal y para las diferentes técnicas atonales. Asimismo, los sistemas de análisis atonal empezaron a tomar fuerza, debido a que los modelos tradicionales no se podían aplicar a las nuevas sonoridades y técnicas composicionales, culminando con la publicación del libro "The Structure of Atonal Music" de Allen Forte ${ }^{4}$, en el cual se plantea el concepto de "Set Theory" (Teoría de Conjuntos) como una herramienta para el análisis y la comprensión de la música atonal.

Por otro lado, las obras de Gutiérrez previas a sus estudios en Estados Unidos eran de un corte completamente tonal tradicional, tal como su ópera Marianela. La tesis realizada por Ronald Ray Sider en el año 1967 es una buena muestra del ámbito nacional en una época posterior a los estudios de Gutiérrez en los Estados Unidos. Dicho período se podría considerar de experimentación y consolidación del estilo composicional de Gutiérrez.

Este período se caracteriza por la utilización de diversas técnicas de vanguardia, las cuales se pueden observar en obras como: "Música para Siete Instrumentos (1965), escrita en Buenos Aires, [Gutiérrez] usa el idioma dodecafónico constantemente, y la Toccata y
Fuga para Piano (1960), escrita bajo supervisión de Milhaud, es muy libre tonalmente, y explora sonoridades disonantes 5 ."

La Sonata para Clarinete y Piano no utiliza el sistema dodecafónico u otras técnicas serialistas, pero las sonoridades son claramente atonales, y existe una clara evasión de la tonalidad. En algunas secciones hay pasajes con referencias tonales, pero están escondidos en la partitura en la forma en la que están escritos, o con la evasión completa de las reglas de la armonía funcional.

La Sonata puede ser considerada atonal, desde el punto de vista que no usa el sistema armónico tradicional como base para establecer las relaciones y un centro tonal, básicamente la relación dominante-tónica. Sin embargo, el compositor restringe los materiales utilizados para generar el contenido melódico y armónico de la obra, estos materiales son limitados a la escala octatónica y otros conjuntos simétricos.

En este movimiento es claro que la escala octatónica es el punto de partida para la construcción de la obra, y la mayoría del material utilizado se puede justificar como subconjuntos de la escala octatónica. Además, el material que es básicamente tonal, puede ser justificado como una interacción con la escala octatónica, debido a las relaciones que se pueden determinar entre la escala octatónica y el sistema tonal. Las referencias y nomenclatura a la escala octatónica son muchas y muy variadas, pero en este estudio se utilizará el sistema basado en Strauss ${ }^{6}$ (OCT0,1 - OCT1,2 - OCT0,2), explicadas en el ejemplo 1 . También se expondrá la posible aplicación de extracción de notas de un pasaje y relacionar esas nota con la escala octatónica, tal como lo utiliza van der Toorn ${ }^{7}$.

El presente trabajo va a discutir los materiales utilizados en la exposición del primer movimiento de la Sonata para Clarinete, de los compases 1 a 23, desde el punto de vista de la relación de las sonoridades con respecto a las diferentes posibilidades de la escala octatónica.

\section{Forma}

Como se puede observar en el ejemplo 2, la pieza está estructurada en una forma 
ABA'Coda. En esta forma, la sección B está compuesta por el desarrollo de los elementos presentes en la sección $\mathrm{A}$. La recapitulación, A', presenta los materiales utilizados durante la exposición, pero con algunas variantes, ya que estos están modificados por medio de una operación $\mathrm{T} 2^{8}$ y además, el material está tratado por medio de contrapunto invertido en las voces del clarinete y la mano derecha del piano a partir del compás 68, como se puede observar en el ejemplo 3. El efecto y el cambio de timbre producto de estas modificaciones, le da a la obra una variedad melódica y una sensación de la presentación de material nuevo, pero a la vez, el intercambio de materiales entre la mano derecha del piano y la parte del clarinete le brinda a la pieza unidad y coherencia.

Esta estructura puede ser entendida como una forma tripartita, con cierta influencia de la forma binaria redondeada, la cual según Miguel Roig-Francolí se puede entender como "un diseño formal (...) el cual presenta el regreso del material inicial de la parte 1 en la segunda sección de la parte $2^{9}$." Sin embargo, el concepto de la forma binaria parte del entendido de una relación armónica de tónica-dominante, lo cual esta obra no posee, por no utilizar relaciones tonales tradicionales.

Otra interpretación de la estructura de esta obra es como una forma ternaria, siguiendo el principio de que una obra es ternaria cuando "está constituída por tres partes cerradas e independientes." En este sentido, la obra se puede apegar a esta estructura, pero el concepto de partes "cerradas" también es una referencia tonal, y como se explicó anteriormente, la noción de tonalidad no se puede aplicar a esta obra. Además, la sección B guarda una estrecha relación de desarrollo de los elementos incluidos en la sección A, por lo que no se puede considerar una parte independiente.

La sección de la Coda, al igual que la sección A', reciclará material presentado anteriormente, ya que en este caso es material incluido en la sección de desarrollo, B, en los compases 23 a 29, los cuales están también modificados por $\mathrm{T} 2 \mathrm{y}$ utilizando contrapunto invertido entre las voces del clarinete y la mano derecha del piano. Al utilizar los mismos materiales que $\mathrm{B}$, podríamos considerar la Coda más bien como una sección B', y en tal caso la forma correspondería a una forma binaria ABA'B'. Sin embargo, la variación en tempo de la última sección es lo que nos lleva a determinar que esta sección es en realidad una Coda, no la sección B'.

En términos generales, esta obra será considerada como una adaptación de la forma binaria redondeada explicada anteriormente. Debido al lenguaje de esta obra no podemos hablar de áreas tonales, sin embargo, el análisis de la obra nos muestra cómo el retorno temático coincide con una transposición del material, lo cual podríamos considerar como una interpretación de la forma, en el lenguaje octatónico. Además, existe claramente una recapitulación, y el tratamiento de los materiales es similar al que se hace dentro de una forma binaria, lo cual es una recapitulación del material en un área tonal diferente.

Sin embargo, aunque la recapitulación inicia en un centro sonoro diferente al de la exposición, no se puede determinar un retorno al centro sonoro original, lo cual acarrea consecuencias formales. El paralelo de esta forma, en una forma binaria tonal, es tener una recapitulación que inicia en la dominante, pero que no regrese a la tónica. En el ámbito tonal, dicha falta de regreso a la tónica conlleva un dilema en la forma, pero al tratarse de un ámbito atonal, y especialmente al trabajar con conjuntos octatónicos, las consecuencias no son determinantes para la estructura de la obra debido a la limitada capacidad de transposición del conjunto octatónico.

\section{Colecciones Octatónicas}

El ejemplo 4 muestra cómo se utilizan las colecciones octatónicas en la exposición del movimiento. Este material, como se dijo anteriormente, será retomado durante la recapitulación, pero modificado por T2. El análisis de los subconjuntos octatónicos utilizados en la recapitulación, por ende, producirá un grupo completamente diferente de conjuntos. 
Sin embargo, al analizar la exposición podemos determinar la interacción de dichos conjuntos y subconjuntos, esta interacción será similar a la que se lleva a cabo durante la recapitulación, con la consecuente modificación T2. Por ejemplo, en la exposición, el compás 1 contiene OCT0,1, el cual será transformado en OCT0,2, por medio de T2 durante la recapitulación. De la misma forma, si aplicamos T2 a OCT1,2, se convertirá en OCT0,1, y a su vez OCT0,2 se convertirá en OCT1,2, tal y como se puede ver en el ejemplo 5.

Dicha interacción de los conjuntos es similar a la de los grados utilizados en una exposición y su correspondencia durante la modulación a la dominante en una forma binaria. El equivalente a esta relación de los conjuntos y subconjuntos de OCT es similar a los procesos de modulación o tonicización, en los cuales, cuando se modula a la dominante, un acorde puede tener una función similar a la del inicio, pero en relación con la dominante.

Un factor importante en el pasaje analizado en este trabajo es que la colección octatónica nunca es presentada en su totalidad. El compositor siempre presenta subconjuntos de la colección, como se puede ver en el ejemplo 6. Si estudiamos la forma primaria de los subconjuntos utilizados en la obra, podemos determinar que la forma más grande derivada de la colección octatónica es el heptacordo 7-31 $(0134679)^{11}$, el cual es utilizado dos veces, en diferentes versiones, una como subconjunto de OCT0,1, y luego como subconjunto de OCT1,2. De la misma manera, el ejemplo 6 muestra como los subconjuntos de OCT son presentados a través de la exposición del primer movimiento.

\section{Simetría y operaciones de generador}

En algunas secciones del fragmento analizado es evidente que los conjuntos no son miembros de la colección octatónica. Sin embargo, dichos conjuntos comparten una característica importante con la colección octatónica, la cual es la simetría.

Es bien sabido que la colección octatónica puede ser generada por medio de una operación TC (Combinación transpositoria, por sus siglas en inglés) ${ }^{12}$, y que hay varias formas de utilizar la operación TC para lograr una colección octatónica. Podemos iniciar utilizando el conjunto 4-28 (0369) y superponer dos de ellos a un semitono de distancia, lo cual se puede explicar como TC1[0,3,6,9] resultando en $[0,1,3,4,6,7,9, t]$. Otra manera de generar OCT podría ser por TC6(TC3[0,1]), como se muestra en el ejemplo 7.

Algunos de los subconjuntos de OCT que aparecen en la obra se pueden explicar de esta manera, y de hecho, esto tiene un efecto en las sonoridades presentes en la obra. Por ejemplo, como se muestra en el ejemplo 6, el conjunto en los compases 2 a 4, es un subconjunto de OCT0,1, el heptacordo 7-31 (013467) presentado como $[\mathrm{t}, 0,1,3,4,6,7]$. En este caso, se puede utilizar una operación TC para generar dicho conjunto, la cual sería TC3(TC3 $[t, 0,1]$ ), como se evidencia en el ejemplo 7. Un procedimiento similar puede ser utilizado para varios conjuntos, tal y como se detalla en el ejemplo 6.

Sin embargo, para algunos de los conjuntos, la operación TC debe incluir inversión, y este es el caso de los compases 1 y 2 , donde el material es un subconjunto de OCT1,2, presentado como el conjunto 6-Z40 (023589), en el orden normal $[1,2,5,7,8, t]$. El ejemplo 9 muestra como en este caso, el conjunto puede ser generado al utilizar [2,5,7], aplicando la operación T3I y combinando el resultado.

Los materiales que no están relacionados a OCT comparten la característica de la simetría. Dichos conjuntos son la escala de tonos enteros, en su versión WT1, un conjunto que resulta como la combinación de HEX con WT1, y el conjunto 8-24.

Un punto interesante se encuentra en los compases 19 y 20 en la mano izquierda del piano, el cual se puede ver desde tres perspectivas diferentes: primero, como una secuencia de cuatro (048); segundo, agrupar las dos primeras tríadas, resultando en HEX y la tercera y cuarta tríadas que resultan en WT1; tercero, agrupar las cuatro tríadas en un conjunto, lo cual significaría añadir HEX y WT1 en un conjunto. Este resultado es el conjunto 9-12 (01245689t), que puede ser fácilmente 
explicado por medio de una operación TC de tres notas cromáticas, o como una operación TC de una tríada aumentada, como se muestra en el ejemplo 10.

El otro conjunto que se utiliza en este fragmento, y que no es parte de OCT, es el conjunto 8-24 (0124568t), presentado en el compás 19 , en la mano derecha del piano y la parte del clarinete. El ejemplo 11 muestra que este conjunto es también simétrico, y se puede explicar por medio de una operación TC con inversión.

El final de la exposición nos presenta un conjunto que puede ser también visto de varias maneras, expuesto en el ejemplo 12. Los compases 21 y 22 presentan el conjunto 6-30 (013679), un subconjunto de OCT0,2, en la forma $[0,2,3,5,8,9]$. Para poder considerar el material de estos compases como parte de este conjunto, debemos explicar el mi natural (4), sol (7) y el si (10) como notas vecinas, o como notas extrañas al conjunto.

Sin embargo, si incluimos dos de esas notas al conjunto, y solo tenemos que justificar el sol como nota vecina al conjunto, entonces se produce el conjunto 8-25.

\section{Fragmentos tonales}

Aunque este análisis se enfoca en las colecciones octatónicas y otros conjuntos relacionados, hay porciones de la exposición que son indiscutiblemente tonales. Uno de estos pasajes se encuentra en los compases 5 a 7, examinado en el ejemplo 13, donde el bajo ejecuta una escala de lab menor, pero finaliza en un movimiento V-I en mib. Las voces extremas apoyan dicho movimiento, pero la resolución del acorde es irregular, ya que las voces internas forman un acorde de la bemol menor en segunda inversión. Básicamente, la resolución es correcta, con la excepción de las voces internas, lo cual resulta en una variación de la cadencia deceptiva.

El otro punto de interés en este sentido es el pasaje en los compases 13 y 14, en el ejemplo 14 , en donde hay una resolución similar a lo que en armonía tradicional se conoce como la sexta aumentada. En este caso, el acorde no cumple con los requisitos de las sextas aumentadas italiana, francesa o alemana, pero las voces siguen las reglas de la resolución de una sexta aumentada: las voces que forma la sexta aumenta resuelven por medio de la apertura a una octava, y las otras voces forman el acorde en posición fundamental. El acorde de resolución en este caso es un acorde de mi mayor, pero escrito con lab (posiblemente para ocultar la referencia tonal de esta resolución). Sin embargo, este procedimiento claramente tonal se desvanece rápidamente por la incorporación de un acorde de mi menor una vez que la resolución a mi mayor es ejecutada.

Como se ha mencionado, el pasaje de los compases 5 a 7 puede ser considerado como un pasaje en lab menor, y de los compases 8 a 11, en lab mayor. La inclusión de dichos pasajes en un trabajo atonal eleva el interés de las posibilidades de la colección octatónica, y el uso de conjuntos simétricos. Una característica interesante del conjunto octatónico es que puede utilizado con referencias tonales, ya que ambas tríadas mayor y menor (037) son subconjunto de OCT, como se puede ver en el ejemplo 15.

\section{Reinterpretación de las áreas estructurales}

Para empezar, el procedimiento por el cual el compositor sale de un área tonal es por medio de la utilización de uno de los conjuntos simétricos más obvios, la escala de tonos enteros. En el compás 11, todas las voces adoptan la escala de tonos enteros como un medio de transición del lenguaje tonal a la sonoridad octatónica. A su vez, es también importante notar que la obra inicia con todas las voces presentando una colección octatónica $(\mathrm{OCT} 1,2)$, pero inmediatamente las voces empiezan a interactuar con las otras opciones de OCT. La única parte en donde todas las voces vuelven a utilizar la misma versión de la colección octatónica, es después de la presentación de la escala de tonos enteros. Desde este punto de vista, podemos relacionar este regreso a OCT como el regreso tonal encontrado en formas binarias o sonata, pero en una forma invertida: 
en este caso OCT está trabajando como el punto de partida, mientras que el área tonal es el punto inestable en la obra, el cual debe ser rápidamente resuelto para regresar al material estable (OCT), tal y como se puede ver en el ejemplo 16.

Esta porción tonal en la exposición podría ser también explicada por medio de OCT. Si seguimos los ejemplos de van den Toorn ${ }^{13}$, podríamos aplicar el mismo concepto que él desarrolla, a dichos pasajes. En el ejemplo 17 vemos cómo se puede relacionar completamente el material del compás 4 a las colecciones octatónicas y a la escala de tonos enteros, así podemos incluso explicar la escala de lab menor armónica por medio de la extracción octatónica.

El ejemplo 18 muestra las posibles relaciones del pasaje en los compases 5 a 7 con OCT, el cual es diatónico en la tonalidad de lab mayor. En este caso, podemos relacionar la escala de lab mayor de alguna forma con cada una de las posibilidades de OCT, pero el pasaje no puede ser relacionado a una sola opción de OCT en su totalidad.

Como se muestra en los ejemplos $17 \mathrm{y}$ 18 , los pasajes tonales pueden relacionarse y ser analizados por medio del uso de las colecciones octatónicas. Sin embargo, dicho análisis no sería de ayuda en determinar las sonoridades generales y el efecto que dichas sonoridades tiene en la pieza. Si bien es cierto que OCT puede estar relacionado a las tríadas mayor y menor como uno de sus posibles subconjuntos, OCT no posee el factor determinante básico de tonalidad, el cual es la dominante.

\section{Elementos melódicos unificadores}

Un detalle que se ha mencionado anteriormente en este breve análisis, es la preponderancia del OCT como sonoridad general en los bloques de la obra, y la utilización de subconjuntos pertenecientes a OCT para construir dichas secciones. Un elemento fácilmente visible es la primacía del subconjunto (016) tanto en forma melódica como armónico a través de la obra. Este subconjunto es la primera figura tanto melódica como armónica de la obra, y también es la que finaliza el movimiento, como se expone en el ejemplo 19.

Este subconjunto representa un elemento de suma importancia en la obra general de Gutiérrez. Como se menciona en el capítulo Rutas para explorar el lenguaje de un compositor, en el apartado de Benjamín Gutiérrez son varias las obras que tienen un inicio similar, y tras un rápido vistazo a dichas composiciones, la gran mayoría de las mencionadas en dicho artículo inician precisamente con el subconjunto (016). Dichas obras son Preludio Sinfónico, Introducción y Allegro (tanto al inicio de Introducción, como en el tema que inicia el fugato), Tramas, Sinfonía Coral (en el tema que inicia el fugato). Las obras expuestas que no se apegan a dicho subconjunto en sus inicios son el Concierto para Viola, y Homenaje a Juan Santamaría.

\section{Conclusión}

Definir el estilo musical de un compositor basado en pocos aspectos musicales es imposible. Por lo tanto, es necesario un análisis profundo y detallado de todas sus obras para poder llegar a vislumbrar las generalidades del estilo de un autor. Con el análisis expuesto en este artículo no se pretende definir el estilo musical de Gutiérrez, pero sí se brindan detalles acerca de la utilización de los elementos musicales del compositor en esta obra.

Las sonoridades utilizadas por Benjamín Gutiérrez en su Sonata para Clarinete y Piano no pueden ser determinadas como tonales. No obstante, la obra no está basada en materiales preconcebidos, ni hay presencia de series u otras técnicas seriales o dodecafónicas. Además, hay muchos factores que unifican la obra, y el hecho de que una forma binaria redondeada ABA' puede ser establecida nos da una idea del tipo de lenguaje y las herramientas analíticas que se pueden utilizar para abordar esta obra.

Después de examinar grandes bloques de material, en vez de examinar conjuntos pequeños y gestos melódicos obvios, la colección octatónica empieza a ser evidente en la textura. Los ejemplos y las tablas presentados en este trabajo demuestran cómo el material 
está directamente relacionado a las colecciones octatónicas y otros conjuntos. Estos conjuntos estudiados comparten la cualidad simétrica de la colección octatónica, por ende, las sonoridades producidas tanto por las colecciones octatónicas así como por los otros conjuntos simétricos va a ser similar, hasta cierto punto.

Las porciones tonales de la obra pueden explicarse como la utilización del sistema tonal como un área inestable en relación con el material base de la obra, el cual es octatónico. De esta forma, se establece una similitud con las formas tradicionales binaria o sonata, en las cuales existe un área inestable con respecto al material base de la obra, el cual es tonal. Sin embargo, este proceso sucede durante la exposición de la obra, el cual se repetirá durante la recapituación, produciendo de esta forma una pequeña estructura ABA dentro de la exposición y la recapitulación.

La forma general de este movimiento es ABA' binaria redondeada, y el uso de técnicas composicionales tradicionales le suma unidad a la obra. Esto, además del uso de la colección octatónica como base para las sonoridades le da a la obra una sensación de estabilidad, aún cuando no es posible establecer centros tonales de importancia en la obra.

Todos los elementos presentados demuestran una clara intencionalidad del compositor en evitar la tonalidad, aunque se presenten remembranzas de los procesos tonales tradicionales. La utilización de ciertos elementos en esta obra marca definitivamente la obra general de Gutiérrez, al punto que el conjunto (016) en una forma melódica al inicio de la obra, se convierte en un elemento unificador que se utiliza en gran cantidad de obras.

El análisis de un fragmento de esta obra expone la necesidad de un estudio serio de obras costarricenses. Este trabajo pretende vislumbrar una muestra del patrimonio musical nacional, a la vez que refleja la utilización de herramientas teórico-musicales necesarias para la comprensión del mismo. La utilización de la teoría de conjuntos demuestra ser una herramienta básica y necesaria para cualquier músico, tanto ejecutante, teórico, musicólogo o de otras ramas de la profesión, para poder entender el contenido de obras de esta índole. Sin la utilización de estas herramientas especializadas, cualquier intento de análisis será superfluo y empírico, contradiciendo las premisas de la investigación académica.

La teoría de conjuntos fue aplicada a la música hace cuarenta años por Allen Forte, tal y como se mencionó. Debido a esto, no se puede considerar como una herramienta de vanguardia, sin embargo, esta ha sido estudiada y utilizada desde entonces para el análisis de obras atonales en los principales centro de estudio e investigación musical. En nuestro país, la situación ha sido diferente, hace pocos años que esta teoría se estudia y aplica en nuestro medio. Sin embargo, son pocas las publicaciones realizadas en las cuales se utilicen estas técnicas. Por tal motivo, es la labor de los investigadores musicales y las casas de publicación nacionales, el promover el estudio del patrimonio musical costarricense bajo los estándares internacionales de análisis musical.

\section{Notas}

1. K. Robert Schwarz, "A Regional Favorite Gains Prominence," New York Times, 1 November 1998.

2. Jacob Druckman, in C. Gagne and T. Caras, Soundpieces: Interviews with American Composers (Metuchen, N.J.: Scarecrow Press, 1982), 156.

3. Michael Beckerman, "Tonality Is Dead, Long Live Tonality,” New York Times, 31 July 1994.

4. Allen Forte, The Structure of Atonal Music. New Haven: Yale University Press, 1973.

5. Ronald Sider, "Contemporary Composers in Costa Rica," Latin American Music Review 5, No. 2, 1984: 268.

6. Strauss, Joseph. Introduction to Post-Tonal Theory, 144-146.

7. Toorn, Pieter van der. The Music of Igor Stravinsky. 
8. La operación Tn se refiere a transporte a un intervalo, siendo $\mathrm{n}$ el intervalo. Para un estudio de esta operación véase Strauss, 39-44.

9. Roig-Francolí, Miguel. Harmony in Context. McGraw Hill, 2003: 589.

10. Ibid, 599.

11. La designación de los conjuntos es de acuerdo con la table Forte. Para más detalle, véase Strauss, 261-264.

12. Para mayor detalle acerca de las operaciones TC, véase Strauss, 98-99.

13. Toorn, Pieter van der. The Music of Igor Stravinsky.

14. Chatski, E., Rutas para explorar los elementos estilisticos del lenguaje musical de un compositor, en Música académica costarricense. Del presente al pasado cercano, por Vargas Cullel, M. C., Chatski, E., Vicente León, T. (2012). San José, SIEDIN, Universidad de Costa Rica.

\section{Bibliografía}

Beckerman, M. 1994, July 31. Tonality Is Dead, Long Live Tonality. The New York Times.

Gagne, C., \& Caras, T. 1982. Soundpieces: Interviews with American Composers. Metuchen, N.J.: Scarecrow Press.
Gutiérrez, B. 1989. Sonata for Bb clarinet and piano. Cherry Hill, NJ: Roncorp.

Roig-Francolí, M. 2003. Harmony in Context. New York, NY: McGraw Hill.

Schwarz, K. R. 1998, November 1. A Regional Favorite Gains Prominence. The New York Times.

Sider, R. R. 1967. The Art Music of Central America - Its Development and Present State. (Unpublished doctoral dissertation) Eastman School of Music, Rochester, NY.

1984. Contemporary Composers in Costa Rica. Latin American Music Review, 5 (2), 263-276.

Strauss, J. 2005. Introduction to Post-Tonal Theory. New Jersey: Prentice Hall.

Toorn, P. C. van den. 1983. The music of Igor Stravinsky. New Haven: Yale University Press.

Vargas Cullel, M. C., Chatski, E., \& Vicente León, T. 2012. Música académica costarricense. Del presente al pasado cercano. San José: SIEDIN, Universidad de Costa Rica.

\section{Anexos}

EJEMPLO 1.

Designación de las escalas octatónicas

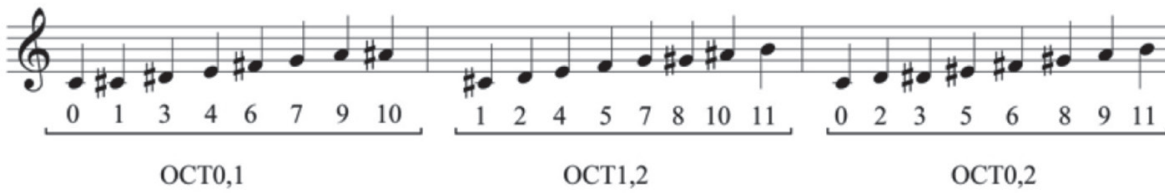


EJEMPLO 2.

Forma binaria redondeada en el primer movimiento de la Sonata para Clarinete y Piano.

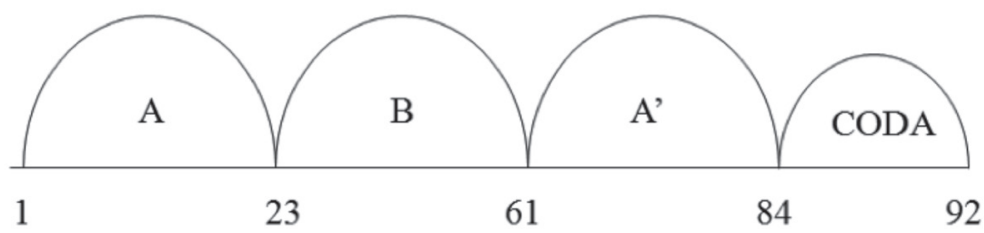

EJEMPLO 3.

Material del inicio de la sección A, compás 1 , y del inicio de la sección A’, compás 62 . El compás 1 se modifica por medio de la operación T2 para general el material del compás $62^{1}$.
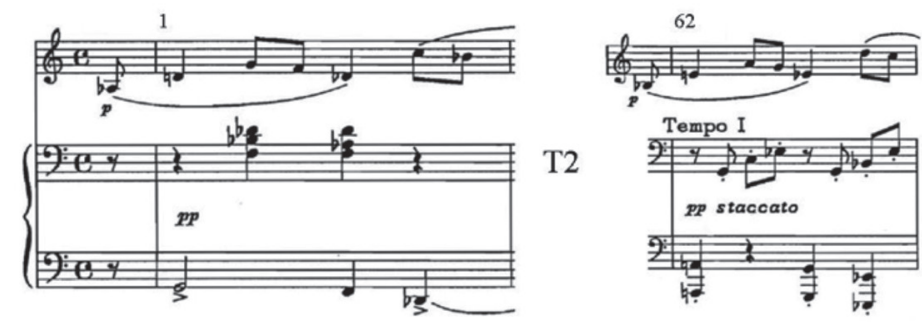

1. Ejemplos musicales utilizados bajo el permiso del editor. La partitura está disponible y puede ser adquirida en Roncorp o Northestern Music Publications, Inc., www.nemusicpub.com.

\author{
EJEMPLO 4
}

Colecciones octatónicas, conjuntos y otros materiales utilizados en la exposición.

\begin{tabular}{|c|c|c|c|c|c|c|c|c|c|c|c|c|c|c|c|c|c|c|c|c|c|c|}
\hline & 1 & 2 & 3 & 4 & 5 & 6 & 7 & 8 & 9 & 10 & 11 & 12 & 13 & 14 & 15 & 16 & 17 & 18 & 19 & 20 & 21 & 22 \\
\hline Clar. & 1,2 & 0,1 & 0,1 & 0,1 & $\mathrm{La}$ & $b$ & $\mathrm{~m}$ & $\mathrm{La}$ & $b$ & M & & WT1 & & & & & & 1,2 & $8-24$ & $6-7$ & 0,2 & 0,2 \\
\hline $\mathrm{MD}$ & 1,2 & 1,2 & & & $\mathrm{La}$ & $b$ & $\mathrm{~m}$ & $\mathrm{La}$ & $b$ & M & & WT1 & 0,1 & 1,2 & 0,2 & 0,2 & 1,2 & 1,2 & $8-24$ & $6-7$ & 0,2 & 0,2 \\
\hline MI & 1,2 & 0,1 & 0,2 & 0,2 & $\mathrm{La}$ & $b$ & $\mathrm{~m}$ & $\mathrm{La}$ & b & M & & WT1 & 0,1 & 1,2 & 1,2 & 1,2 & 0,1 & 0,1 & HEX & WT1 & 0,2 & 0,2 \\
\hline
\end{tabular}


EJEMPLO 5

Resultado de la operación T2 en OCT

OCT0,1 $[0,1,3,4,6,7,9, \mathrm{t}] \mathrm{T} 2=$ OCT0,2 $[0,2,3,5,6,8,9, \mathrm{e}]$

OCT1,2 $[1,2,4,5,7,8, \mathrm{t}, \mathrm{e}] \mathrm{T} 2=\mathrm{OCT} 0,1[0,1,3,4,6,7,9, \mathrm{t}]$

OCT0,2 $[0,2,3,5,6,8,9, \mathrm{e}] \mathrm{T} 2=\mathrm{OCT} 1,2[1,2,4,5,7,8, \mathrm{t}, \mathrm{e}]$

EJEMPLO 6

Conjuntos utilizados en la exposición.

\begin{tabular}{|c|c|c|c|c|c|c|}
\hline Colección & Compases & Sub-Colección & N.O. & Conjunto & Forte & $\begin{array}{l}\text { Operación } \\
\text { Generadora }\end{array}$ \\
\hline$\overline{\mathrm{OCT}}$ & $2-4$ & \multirow{3}{*}{ OCT 0,1} & {$[\mathrm{t}, 0,1,3,4,6,7]$} & $(0134679)$ & $7-31$ & TC3(TC3[t, 0,1$])$ \\
\hline$(0134679 t)$ & 13 & & {$[7, \mathrm{t}, 0,1]$} & $(0136)$ & $4-13$ & \\
\hline \multirow[t]{9}{*}{$8-28$} & $17-18$ & & {$[\mathrm{t}, 0,1,4,7]$} & (01369) & $5-31$ & \\
\hline & $14-16$ & \multirow{3}{*}{ OCT 1,2} & {$[2,4,5,7,8, \mathrm{t}, \mathrm{e}]$} & $(0134679)$ & $7-31$ & TC3(TC3[2,4,5]) \\
\hline & $1-2$ & & {$[1,2,5,7,8, \mathrm{t}]$} & (023589) & $6-Z 50$ & T3I $[2,5,7]$ \\
\hline & $17-18$ & & {$[5,7,8, \mathrm{t}, 1]$} & $(02358)$ & $5-25$ & \\
\hline & $3-4$ & \multirow[b]{2}{*}{ OCT 0,2} & {$[3,5,6,8,9]$} & $(01346)$ & $5-10$ & TC3[3,5,6] \\
\hline & $15-16$ & & {$[\mathrm{e}, 0,2,3,5,8]$} & (013469) & $6-27$ & \\
\hline & $21-22$ & OCT 0,2 & {$[0,2,3,6,8,9]$} & $(013679)$ & $6-30$ & TC6[0,2,3] \\
\hline & & $\begin{array}{l}\text { (-mi natural -sol -sib, } \\
\text { tonos vecinos) }\end{array}$ & & & & \\
\hline & & $\begin{array}{l}\text { ó OCT } 0,2+\mathrm{mi}+\mathrm{sib} \\
(\text { sol siempre vecino) }\end{array}$ & {$[2,3,4,6,8,9, \mathrm{t}, 0]$} & $(0124678 t)$ & $8-25$ & TC6 $[0,2,3,4]$ \\
\hline$\overline{\mathrm{WT}}$ & 12 & WT1 & {$[1,3,5,7,9, \mathrm{e}]$} & $(02468 t)$ & $6-35$ & $\mathrm{C} 2-1$ \\
\hline$\overline{\mathrm{HEX}+\mathrm{WT} 1}$ & $19-20$ & & {$[3,4,5,7,8,9, \mathrm{e}, 0,1]$} & $(01245689 t)$ & $9-12$ & $\begin{array}{l}\text { TC8(TC4[3,4,5]) } \\
\text { TC1(TC1 }[3,7, \mathrm{e}])\end{array}$ \\
\hline $8-24$ & 19 & & {$[3,4,5,7,8,9, \mathrm{e}, 1]$} & $(0124568 t)$ & $8-24$ & T0I[1,3,4,5] \\
\hline 6-7 (012478) & 20 & & {$[1,2,3,7,8,9]$} & $(012678)$ & $6-7$ & $\begin{array}{l}\text { TC6[123] } \\
\text { T8I }[1,2,7]\end{array}$ \\
\hline
\end{tabular}


EJEMPLO 7

Operaciones TC como medio de generar OCT

$$
\begin{gathered}
\text { TC1 }[0,3,6,9] . \mathrm{T} 1[0,3,6,9]=[1,4,7, \mathrm{t}] .[0,3,6,9]+[1,4,7, \mathrm{t}]=[0,1,3,4,6,7,9, \mathrm{t}] \\
\mathrm{TC} 6(\mathrm{TC} 3[0,1]) . \mathrm{T} 3[0,1]=[3,4] .[0,1]+[3,4]=[0,1,3,4] . \mathrm{T} 6[0,1,3,4]= \\
{[6,7,9, \mathrm{t}] .[0,1,3,4]+[6,7,9, \mathrm{t}]=[0,1,3,4,6,7,9, \mathrm{t}]}
\end{gathered}
$$

EJEMPLO 8

Operación TC como medio generador del conjunto 7-31

$$
\begin{aligned}
& 7-31=\mathrm{TC} 3(\mathrm{TC} 3[\mathrm{t}, 0,1]) \\
& {[\mathrm{t}, 0,1] \text { TC3 }[\mathrm{t}, 0,1,1,3,4]}
\end{aligned}
$$

$[\mathrm{t}, 0,1,3,4] \mathrm{TC} 3[\mathrm{t}, 0,1,3,4,1,3,4,6,7]=[\mathrm{t}, 0,1,3,4,6,7]$

EJEMPLO 9

Conjunto 6-Z40 (023589) en el orden normal [1,2,5,7,8,t], generado por medio de una operación TnI.

$$
[2,5,7] \mathrm{T} 3 \mathrm{I}=[8, \mathrm{t}, 1] .[2,5,7]+[8, \mathrm{t}, 1]=[1,2,5,7,8, \mathrm{t}]
$$

EJEMPLO 10

Posibles construcciones del conjunto 9-12.

$\operatorname{HEX}[3,4,7,8, \mathrm{e}, 0]+\mathrm{WT} 1[1,3,5,7,9, \mathrm{e}]=[3,4,5,7,8,9, \mathrm{e}, 0,1]$

$\operatorname{TC} 8(\mathrm{TC} 4[3,4,5])$ о $\mathrm{TC} 1(\mathrm{TC} 1[3,7, \mathrm{e}])$

EJEMPLO 11

Operación TnI que general el conjunto 8-24 [3,4,5,7,8,9,e,1]

$$
[1,3,4,5] \text { T0I }[7,8,9, \mathrm{e}]=[3,4,5,7,8,9, \mathrm{e}, 1]
$$

EJEMPLO 12.

Compases 21 y 22 .

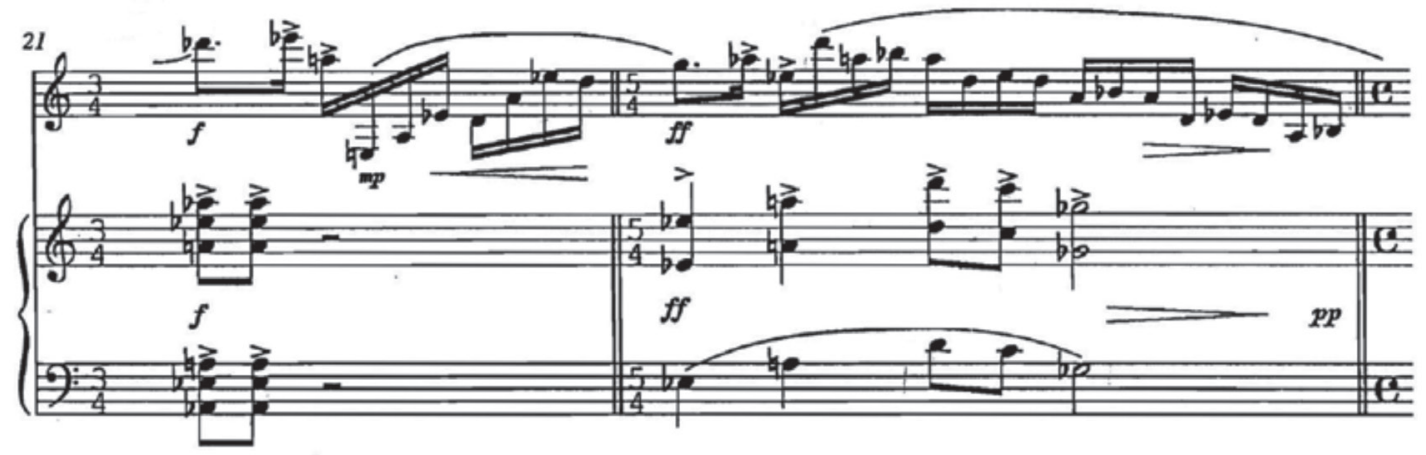


EJEMPLO 13

Compases 5 a 7 .

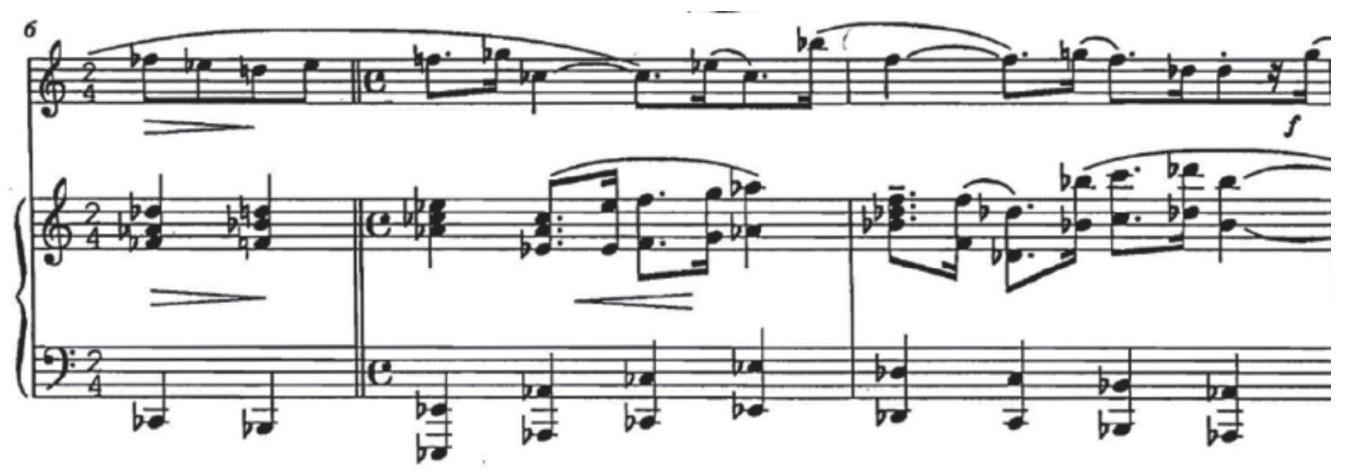

EJEMPLO 14

Compases 13 y 14 .

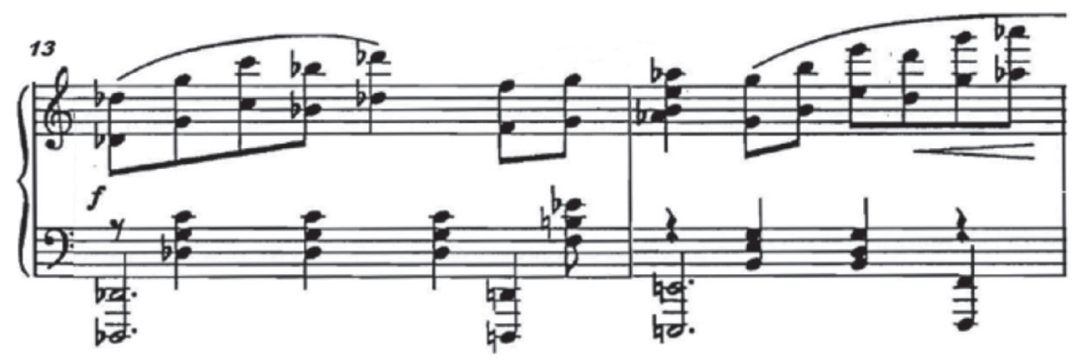


EJEMPLO 15

Tríadas mayores $\left(\mathrm{do}\right.$, la $\mathrm{re}_{\sharp}$, la, fa $\mathrm{fa}_{\sharp}$ como subconjuntos de OCT0,1.Do mayor

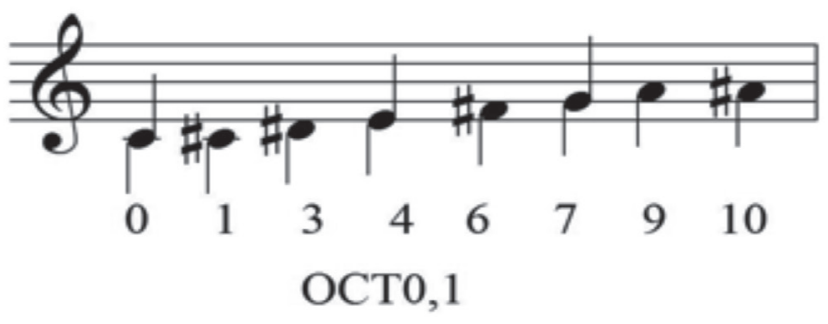

La mayor

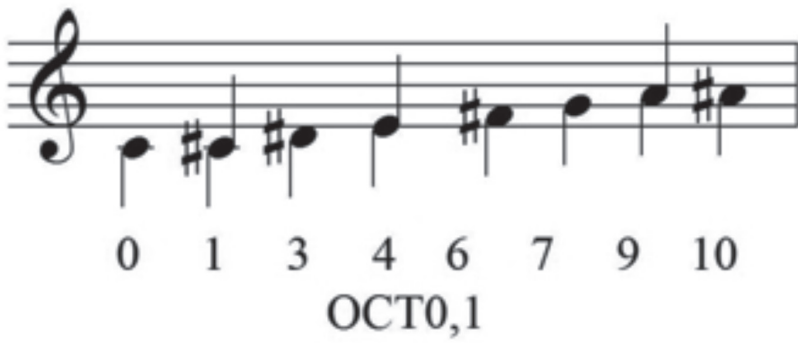

Re\# mayor (sol enarmónico a fax)

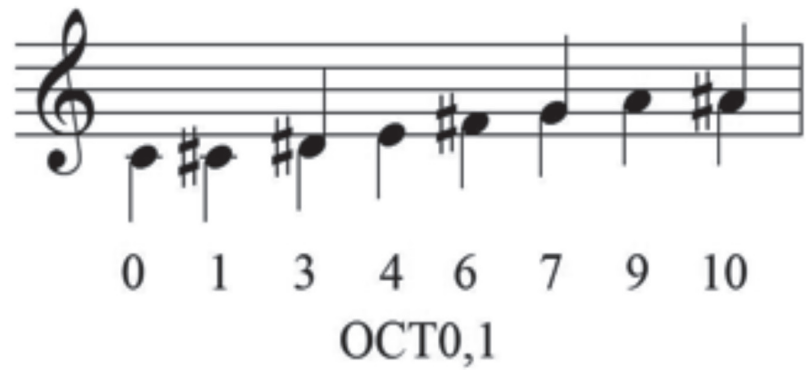


Fa mayor

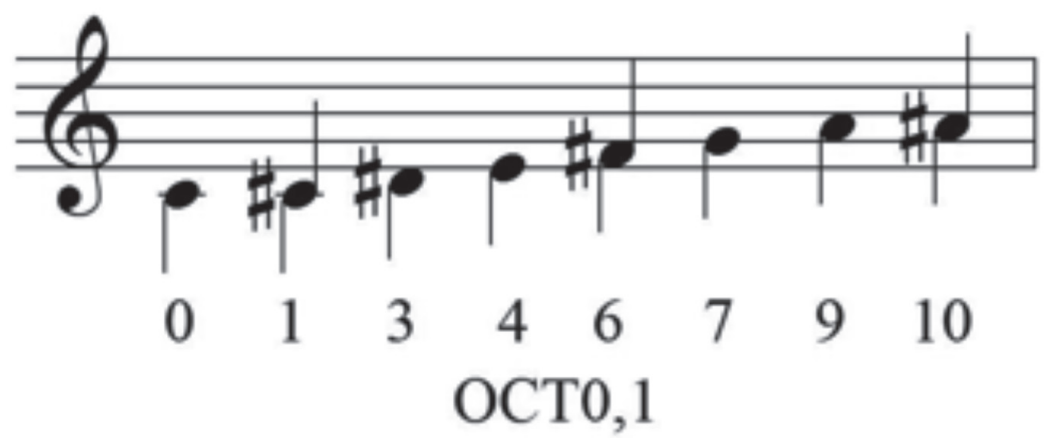

EJEMPLO 16

Reinterpretación de las áreas formales

\begin{tabular}{|c|c|c|c|c|c|c|c|c|c|c|c|c|c|c|c|c|c|c|c|c|c|c|}
\hline & 1 & 2 & 3 & 4 & 5 & 6 & 7 & 8 & 9 & 10 & 11 & 12 & 13 & 14 & 15 & 16 & 17 & 18 & 19 & 20 & 21 & 22 \\
\hline Clar. & 1,2 & 0,1 & 0,1 & 0,1 & $\mathrm{La}$ & b & $\mathrm{m}$ & $\mathrm{La}$ & $b$ & $\mathrm{M}$ & & WT1 & & & & & & 1,2 & $8-24$ & $6-7$ & 0,2 & 0,2 \\
\hline MD & 1,2 & 1,2 & & & $\mathrm{La}$ & $b$ & $\mathrm{~m}$ & $\mathrm{La}$ & $b$ & M & & WT1 & 0,1 & 1,2 & 0,2 & 0,2 & 1,2 & 1,2 & $8-24$ & $6-7$ & 0,2 & 0,2 \\
\hline MI & 1,2 & 0,1 & 0,2 & 0,2 & $\mathrm{La}$ & , & $\mathrm{m}$ & $\mathrm{La}$ & $b$ & $\mathrm{M}$ & & WT1 & 0,1 & 1,2 & 1,2 & 1,2 & 0,1 & 0,1 & HEX & WT1 & 0,2 & 0,2 \\
\hline & $\mathrm{O}$ & $\mathrm{C}$ & $T$ & & $\mathbf{T}$ & $\mathrm{O}$ & $\mathbf{N}$ & A & $\mathbf{L}$ & & & WT & & O & C & $\mathbf{T}$ & & & & & & \\
\hline
\end{tabular}


EJEMPLO 17

Posibles relaciones de OCT en el compás 5.

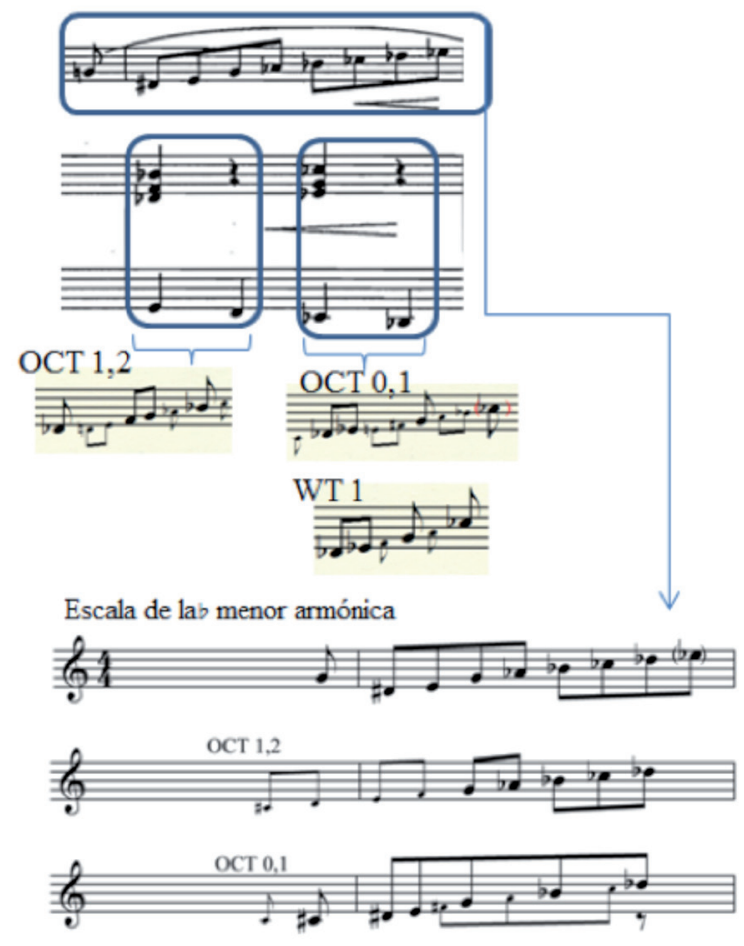

EJEMPLO 18

Compases 9 a 11. Lab mayor y su relación con OCT.

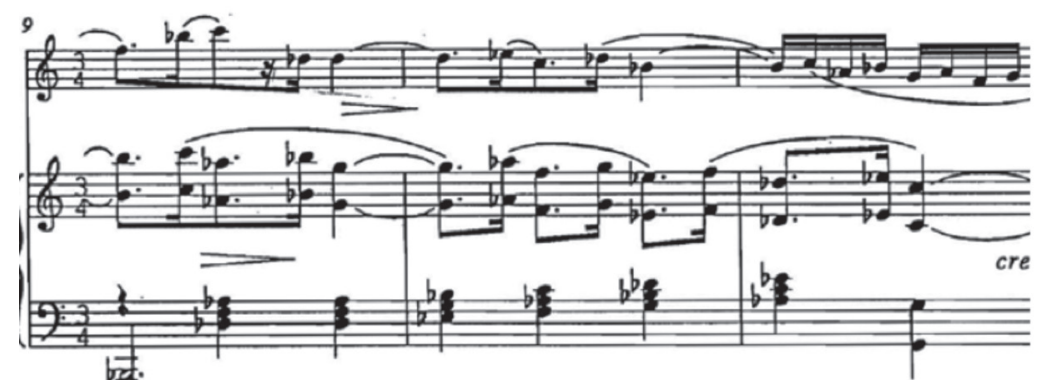



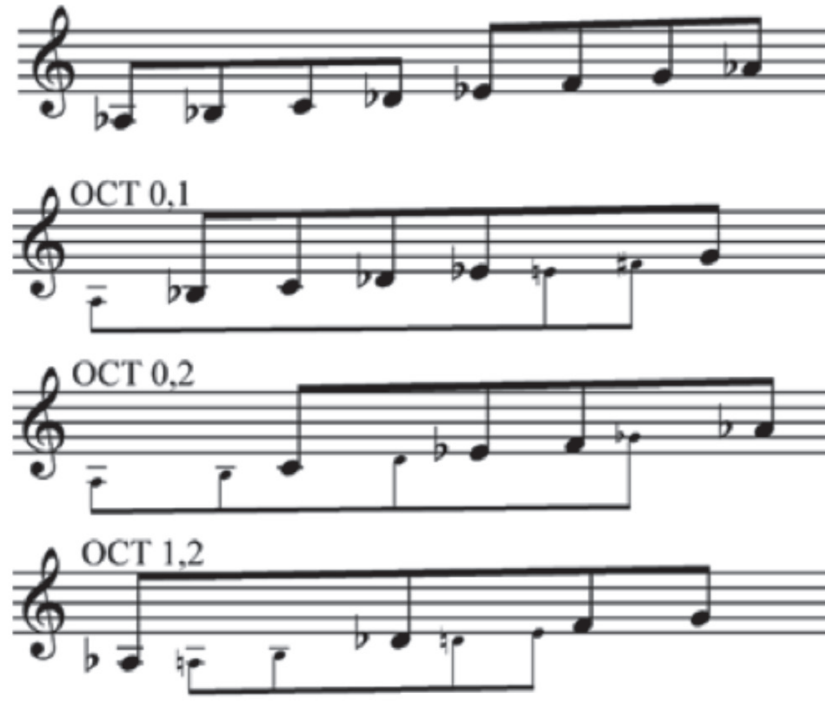

EJEMPLO 19

Gestos inicial y final de la obra, utilizando el conjunto (016)

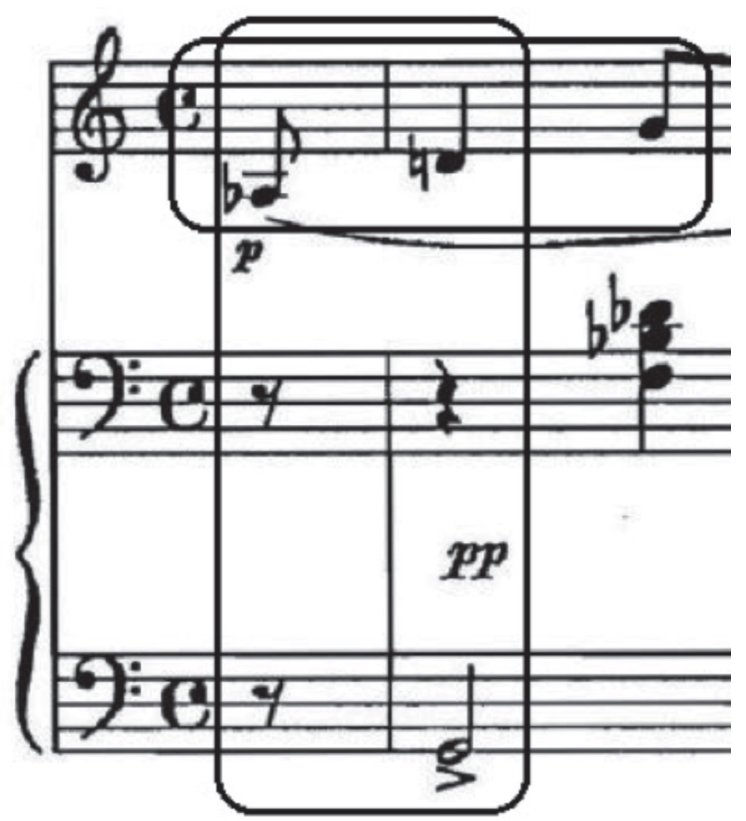


Gesto final

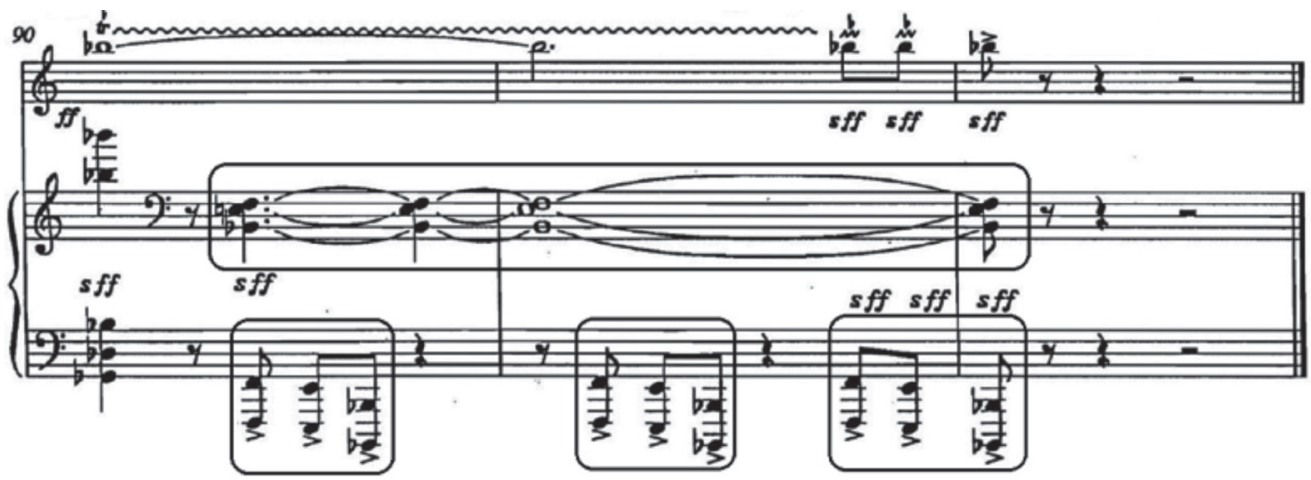


